\title{
Hemoglobin Corrected Diffusion Capacity of the Lung for Carbon Monoxide/Alveolar Volume Ratio
}

National Cancer Institute

\section{Source}

National Cancer Institute. Hemoglobin Corrected Diffusion Capacity of the Lung for

Carbon Monoxide/Alveolar Volume Ratio. NCI Thesaurus. Code C120827.

The diffusion capacity of the lungs for carbon monoxide adjusted for the ratio of hemoglobin concentration to alveolar volume. 